\title{
Five novel species of the genus Nocardiopsis isolated from hypersaline soils and emended description of Nocardiopsis salina Li et al. 2004
}

\author{
Correspondence \\ Wen-Jun Li \\ wjli@ynu.edu.cn \\ Chang-Jin Kim \\ changjin@kribb.re.kr
}

\author{
Wen-Jun Li, ${ }^{1}$ Reiner M. Kroppenstedt, ${ }^{2}$ Dong Wang, ${ }^{1,3}$ Shu-Kun Tang, ${ }^{1}$ \\ Jae-Chan Lee, ${ }^{4}$ Dong-Jin Park, ${ }^{4}$ Chang-Jin Kim, ${ }^{4} \mathrm{Li}-\mathrm{Hua} \mathrm{Xu}^{1}$ \\ and Cheng-Lin Jiang ${ }^{1}$
}

\author{
${ }^{1}$ Laboratory for Conservation and Utilization of Bio-Resources, Yunnan Institute of Microbiology, \\ Yunnan University, Kunming, Yunnan, 650091, PR China \\ ${ }^{2} \mathrm{DSMZ}$ - Deutsche Sammlung von Mikroorganismen und Zellkulturen GmbH, Mascheroder \\ Weg 1b, D-38124 Braunschweig, Germany \\ ${ }^{3}$ Science College, Honghe University, MengZi, Yunnan, 661100, PR China \\ ${ }^{4}$ Korea Research Institute of Bioscience and Biotechnology. Daejeon 305-333, Korea
}

Five novel Nocardiopsis strains isolated from hypersaline soils in China were subjected to a polyphasic analysis to determine their taxonomic position. All of the novel isolates could grow on agar plates at $\mathrm{NaCl}$ concentrations of up to $18 \%(\mathrm{w} / \mathrm{v})$, with optimum growth at $5-8 \%$. The DNA G $+C$ contents of the novel strains ranged from 67.9 to $73.2 \mathrm{~mol} \%$. The morphological and chemotaxonomic characteristics of the isolates matched those described for members of the genus Nocardiopsis. Based on their 16S rRNA gene sequence analysis, DNA-DNA hybridization values and phenotypic characteristics, including the composition of cell-wall amino acids and sugars, menaquinones, polar lipids and cellular fatty acids, the isolates are proposed as representing five novel species of the genus Nocardiopsis. The novel species are proposed as Nocardiopsis gilva sp. nov. [type strain YIM $90087^{\top}\left(=\right.$ KCTC $19006^{\top}=$ CCTCC AA $\left.2040012^{\top}=\mathrm{DSM} 44841^{\top}\right)$ ], Nocardiopsis rosea sp. nov. [type strain YIM $90094^{\top}(=\mathrm{KCTC}$ $19007^{\top}=$ CCTCC AA $2040013^{\top}=$ DSM $44842^{\top}$ ), Nocardiopsis rhodophaea sp. nov. [type strain YIM $90096^{\top}\left(=\right.$ KCTC $19049^{\top}=$ CCTCC AA $2040014^{\top}=$ DSM $\left.44843^{\top}\right)$, Nocardiopsis chromatogenes sp. nov. [type strain YIM $90109^{\top}\left(=\right.$ KCTC $19008^{\top}=$ CCTCC AA $2040015^{\top}=\mathrm{DSM} 44844^{\top}$ ) and Nocardiopsis baichengensis sp. nov. [type strain YIM $90130^{\top}$ $\left(=\right.$ KCTC $19009^{\top}=$ CCTCC AA $2040016^{\top}=$ DSM $\left.44845^{\top}\right)$. On the basis of the chemotaxonomic data, the description of the recently described species Nocardiopsis salina Li et al. 2004 is emended.
The genus Nocardiopsis was first described by Meyer (1976) and, at present, the genus comprises 19 species with validly published names, including several recently described species (Hozzein et al., 2004; Li et al., 2004; Sabry et al., 2004). Members of the genus Nocardiopsis have been reported to predominate in saline or alkaline soils (Tang et al., 2003) and several recognized species have been isolated from such

The GenBank/EMBL/DDBJ accession numbers for the 16S rRNA gene sequences of strains ${\mathrm{YIM} 90087^{\top} \text {, YIM 90094 }}^{\top}$, YIM 90096 ${ }^{\top}$, YIM 90109 ${ }^{\top}$ and YIM 90130 ${ }^{\top}$ are AY619712-AY619716, respectively.

Scanning electron micrographs of the five novel strains and tables detailing their polar lipid profiles, menaquinone patterns, fatty acid contents and DNA-DNA relatedness are available as supplementary material in IJSEM Online. sources (Al-Tai \& Ruan, 1994; Chun et al., 2000; Al-Zarban et al., 2002; Li et al., 2003; Hozzein et al., 2004; Li et al., 2004).

During a taxonomic study of extremophilic actinomycetes, more than 200 Nocardiopsis-like strains were isolated from hypersaline soils in Xinjiang Province, China, and analysed on the basis of their morphology and chemotype. In this paper, we report the phenotypic and genotypic characteristics of five novel isolates and it is proposed that they represent five novel species of the genus Nocardiopsis.

Strains YIM 90087 ${ }^{\mathrm{T}}$, YIM $90094^{\mathrm{T}}$, YIM $90096^{\mathrm{T}}$, YIM $90109^{\mathrm{T}}$ and YIM $90130^{\mathrm{T}}$ were isolated from saline soil samples by using modified International Streptomyces Project (ISP) ISP5 medium supplemented with $15 \% \mathrm{NaCl}$ (w/v). These 
strains were maintained on ISP5 or potato agar slants containing $5 \% \mathrm{NaCl}(\mathrm{w} / \mathrm{v})$ at $4{ }^{\circ} \mathrm{C}$ and as $20 \%(\mathrm{w} / \mathrm{v})$ glycerol suspensions at $-20{ }^{\circ} \mathrm{C}$. Biomass for chemical and molecular systematic studies was obtained by cultivation for 1 week in shake flasks (about 150 r.p.m.) using modified ISP5 medium $(5 \% \mathrm{NaCl}, \mathrm{w} / \mathrm{v}$; $\mathrm{pH} 7 \cdot 0)$ broth at $30^{\circ} \mathrm{C}$ (for strain YIM $90087^{\mathrm{T}}$ ) or $37^{\circ} \mathrm{C}$ (for strains YIM $90094^{\mathrm{T}}$, YIM $90096^{\mathrm{T}}$, YIM $90109^{\mathrm{T}}$ and YIM $90130^{\mathrm{T}}$ ). Additionally, the recently described species Nocardiopsis salina YIM $90010^{\mathrm{T}}$ (Li et al., 2004) was re-examined using standard DSMZ chemotaxonomic methods.

Morphological characteristics of the five novel strains were observed by light microscopy (BH 2; Olympus) and scanning electron microscopy (JSM5600LV; JEOL) after 21 days growth on potato agar medium containing $5 \% \mathrm{NaCl}(\mathrm{w} / \mathrm{v})$. Cultural characteristics were determined after 2-3 weeks by methods used in the ISP (Shirling \& Gottlieb, 1966). All media were supplemented with $5 \% \mathrm{NaCl}(\mathrm{w} / \mathrm{v})$ and the colours of substrate and aerial mycelia and any soluble pigments produced were determined by comparison with chips from the ISCC-NBS colour charts (Kelly, 1964) (see Table 1).

For all five novel strains, vegetative hyphae were well developed and fragmented. Spore chains were borne on aerial hyphae and spores were non-motile. For strains YIM $90087^{\mathrm{T}}$, YIM $90094^{\mathrm{T}}$ and YIM $90096^{\mathrm{T}}$, aerial hyphae did not develop very well on most of the media tested and their spore chains were short. For strains YIM $90109^{\mathrm{T}}$ and YIM $90130^{\mathrm{T}}$, aerial hyphae developed well on most media tested and their spore chains were long (see Supplementary Fig. S1a-j in IJSEM Online).

Media and procedures used for physiological and biochemical features and carbon source utilization were as described by Shirling \& Gottlieb (1966) and Locci (1989). The results are indicated in Table 2 or in the species descriptions.

Amino acid and sugar analysis of cell walls was conducted according to the procedures described by Hasegawa et al. (1983). Polar lipids were extracted, examined by twodimensional TLC and identified using previously described procedures (Minnikin et al., 1984). Menaquinones were isolated according to Minnikin et al. (1984) and separated by HPLC (Kroppenstedt, 1982). Cellular fatty acid analysis was performed as described by Sasser (1990) using the Microbial Identification System (MIDI). All five novel strains, together with strain YIM $90010^{\mathrm{T}}$, contained mesodiaminopimelic acid as the diagnostic diamino acid in the cell wall; no diagnostic sugars were observed. The phospholipids comprised phosphatidylmethylethanolamine (PME), phosphatidylcholine (PC), phosphatidylinositol (PI), phosphatidylglycerol (PG) and diphosphatidylglycerol (DPG), together with some unknown phosphoglycolipids (PGL) and unknown phospholipids (PL). Strains YIM 90096 ${ }^{\mathrm{T}}$, YIM $90109^{\mathrm{T}}$ and YIM $90130^{\mathrm{T}}$ also contained phosphatidylinositolmannosides (PIM). Many differences were found between the six strains in their menaquinone and fatty acid characteristics. Detailed results of the analyses are given in Supplementary Tables S1-S3 in IJSEM Online.

Extraction of genomic DNA and amplification of the 16S rRNA genes were performed as described by Cui et al. (2001) and Xu et al. (2003). Phylogenetic analyses were performed using the PHYLIP (Felsenstein, 1993) and MEGA version 2.1 (Kumar et al., 2001) software packages after multiple alignment of data by CLUSTAL_X (Thompson et al., 1997). Distances, with distance options according to the Kimura twoparameter model (Kimura, 1980, 1983), and clustering were performed using the neighbour-joining method (Saitou \& Nei, 1987). Bootstrap analysis was used to evaluate the tree topology of the neighbour-joining data by performing 1000 resamplings (Felsenstein, 1985).

The method of Marmur (1961) was used to prepare genomic DNA of the five novel isolates for the analysis of base composition. The DNA G $+\mathrm{C}$ contents were determined using the thermal denaturation method of Marmur \& Doty (1962). The DNA G +C contents of the genomic DNAs from strains YIM $90087^{\mathrm{T}}$, YIM $90094^{\mathrm{T}}$, YIM $90096^{\mathrm{T}}$, YIM $90109^{\mathrm{T}}$ and YIM $90130^{\mathrm{T}}$ were $68 \cdot 1,67 \cdot 9,69 \cdot 0,71 \cdot 8$ and $73.2 \mathrm{~mol} \%$, respectively.

16S rRNA gene sequences of the five novel isolates ranged between $1438 \mathrm{bp}$ and $1490 \mathrm{bp}$. Preliminary comparison of the sequences against the GenBank database indicated that the five novel isolates were closely related to members of the genus Nocardiopsis and were most closely related to Nocardiopsis halophila and Nocardiopsis composta. Phylogenetic analyses showed that the five novel isolates fall into one distinct clade with the type strains of $N$. halophila DSM $44494^{\mathrm{T}}$ and N. composta DSM $44551^{\mathrm{T}}$ (Fig. 1). Furthermore, three of the novel isolates, YIM $90087^{\mathrm{T}}$, YIM $90094^{\mathrm{T}}$ and YIM $90096^{\mathrm{T}}$, formed a distinct subclade. The two other novel isolates formed a distinct subclade with $N$. halophila DSM $44494^{\mathrm{T}}$. The five novel isolates showed low $16 \mathrm{~S}$ rRNA gene sequence similarities (below $96 \%$ ) with other recognized Nocardiopsis species, except for $N$. composta $(96 \cdot 4-$ $97 \cdot 5 \%$ similarity) and N. halophila (96.1-99.9\%). The $16 \mathrm{~S}$ rRNA gene sequence similarity between the five novel strains was between 95.9 and $98.7 \%$ (detailed information on gene sequence similarity values between the five novel isolates and their two closest neighbours is presented in Supplementary Table S4 in IJSEM Online). However, high degrees of $16 \mathrm{~S}$ rRNA gene sequence similarity ( $97 \%$ and higher) have been demonstrated to be of limited value for differentiating species and DNA-DNA hybridization studies need to be performed to determine species affiliation (Stackebrandt \& Goebel, 1994).

Accordingly, DNA-DNA hybridization studies were performed using the optical renaturation method (De Ley et al., 1970; Huß et al., 1983; Jahnke, 1992) between the five novel isolates and N. halophila DSM $44494^{\mathrm{T}}$ and N. composta DSM $44551^{\mathrm{T}}$. DNA-DNA relatedness values ranged from 0 to $55.9 \%$ (tests were repeated twice and the mean values are shown in Supplementary Table S4 in IJSEM Online). All of 
Table 1. Culture characteristics of the five novel strains

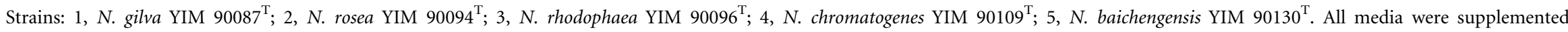

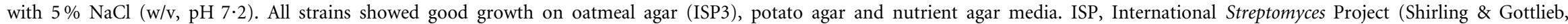
1966). Only strain YIM $90109^{\mathrm{T}}$ produced diffusible yellowish pink pigment on Czapek agar and ISP5 media, etc. Colours are taken from ISCC-NBS colour charts (Kelly, 1964).

\begin{tabular}{|c|c|c|c|c|c|}
\hline Medium & 1 & 2 & 3 & 4 & 5 \\
\hline \multicolumn{6}{|l|}{ Czapek agar } \\
\hline Growth & Good & Poor & Good & Good & Good \\
\hline Aerial mycelium & Pale yellow & Pink-white & Pale pink & White & White \\
\hline Substrate mycelium & Pale yellow & Pale pink & Light reddish brown & Moderate reddish brown & Light yellow \\
\hline \multicolumn{6}{|c|}{ Glycerol-asparagine agar (ISP5) } \\
\hline Growth & Poor & Poor & Good & Good & Good \\
\hline Aerial mycelium & Soft yellow & Grey-pink & Light reddish orange & White & Yellow-white \\
\hline Substrate mycelium & Moderate yellow & Moderate reddish pink & Soft reddish brown & Soft brown & Moderate orange-yellow \\
\hline \multicolumn{6}{|c|}{ Inorganic salts-starch agar (ISP4) } \\
\hline Growth & Good & Good & Good & Poor & Poor \\
\hline Aerial mycelium & Yellow-white & Pink-white & Pale yellow-pink & White & White \\
\hline Substrate mycelium & Pale greenish yellow & Pale pink & Grey-reddish brown & Light reddish brown & White \\
\hline \multicolumn{6}{|c|}{ Yeast extract-malt extract (ISP2) } \\
\hline Growth & Good & Good & Good & Good & Poor \\
\hline Aerial mycelium & Pale yellow & Pale pink & Pale pink & White & Yellow-white \\
\hline Substrate mycelium & Brilliant yellow & Moderate pink & Deep reddish brown & Deep reddish brown & Deep orange-yellow \\
\hline \multicolumn{6}{|l|}{ Oatmeal agar (ISP3) } \\
\hline Aerial mycelium & Yellow-white & Pale pink & Light reddish brown & White & Yellow-white \\
\hline Substrate mycelium & Pale yellow & Moderate red & Grey-reddish orange & Brown & Deep yellow \\
\hline \multicolumn{6}{|l|}{ Potato agar } \\
\hline Aerial mycelium & Yellow-white & Moderate reddish brown & Pale pink & White & Yellow-white \\
\hline Substrate mycelium & Soft yellow & Deep red & Deep reddish brown & Reddish brown & Deep orange-yellow \\
\hline \multicolumn{6}{|l|}{ Nutrient agar } \\
\hline Aerial mycelium & Pale yellow & Pale pink & Pale pink & White & White \\
\hline Substrate mycelium & Pale yellow & Moderate red & Soft reddish brown & Light reddish brown & Pale yellow \\
\hline
\end{tabular}


Table 2. Phenotypic characteristics that differentiate the five novel isolates and the two most closely related Nocardiopsis species

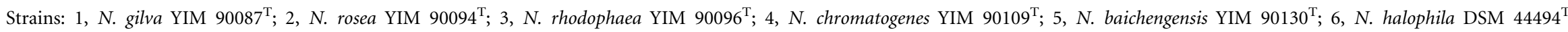

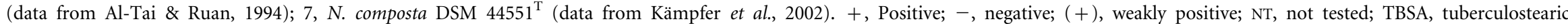
acid, 10-methyl $\mathrm{C}_{18: 0}$.

\begin{tabular}{|c|c|c|c|c|c|c|c|}
\hline Characteristic & 1 & 2 & 3 & 4 & 5 & 6 & 7 \\
\hline Spore chains & Short & Short & Short & Long & Long & Long & Long \\
\hline Colony pigmentation (ISP5) & Pale yellow & Pale pink & Pale pink & White & White & White & White \\
\hline Temperature range for growth $\left({ }^{\circ} \mathrm{C}\right)$ & $10-40$ & $20-60$ & $20-60$ & $20-60$ & $20-50$ & $15-36$ & $15-50$ \\
\hline Optimum temperature for growth $\left({ }^{\circ} \mathrm{C}\right)$ & $28-30$ & $37-40$ & $37-40$ & $37-40$ & $37-40$ & 30 & 37 \\
\hline $\mathrm{NaCl}$ range for growth (\%) & $0-18$ & $0-18$ & $0-18$ & $0-18$ & $0-18$ & $3-20$ & $0-15$ \\
\hline Optimum $\mathrm{NaCl}$ concentration (\%) & $5-8$ & $5-8$ & $5-8$ & $5-8$ & $5-8$ & $5-15$ & 10 \\
\hline Starch hydrolysis & - & - & - & + & - & NT & NT \\
\hline $\mathrm{H}_{2} \mathrm{~S}$ production & - & - & - & - & - & NT & NT \\
\hline Nitrate reduction & + & - & - & - & - & NT & NT \\
\hline Gelatin liquefaction & - & - & - & - & + & NT & NT \\
\hline Melanin production & - & - & - & + & + & NT & - \\
\hline \multicolumn{8}{|l|}{ Carbon source utilization: } \\
\hline D-Fructose & + & + & - & + & + & NT & + \\
\hline D-Galactose & + & - & - & + & + & + & + \\
\hline myo-Inositol & + & - & + & + & - & + & + \\
\hline D-Maltose & - & + & - & + & + & + & - \\
\hline D-Mannose & - & - & - & + & + & NT & + \\
\hline L-Rhamnose & - & + & - & + & + & $(+)$ & - \\
\hline D-Ribose & - & + & + & + & + & NT & + \\
\hline D-Sucrose & + & + & - & + & + & + & - \\
\hline D-Xylose & + & - & - & + & - & + & - \\
\hline \multicolumn{8}{|l|}{ Chemical characteristic: } \\
\hline Polar lipids & $\begin{array}{c}\text { PIII*; DPG, PC, }^{*} \text { PI, PG, PME, } \\
\text { PE, PL, GL }\end{array}$ & $\begin{array}{l}\text { PIII; DPG, PC, } \\
\text { PI, PG, PME, } \\
\text { PE, PL, GL }\end{array}$ & $\begin{array}{l}\text { PIII; DPG, PC, } \\
\text { PI, PG, PME, PE, } \\
\text { PL, GL, PIM }\end{array}$ & $\begin{array}{l}\text { PIII; DPG, PC, } \\
\text { PI, PG, PME, } \\
\text { PE, PL, GL, PIM }\end{array}$ & $\begin{array}{l}\text { PIII; DPG, PC, } \\
\text { PI, PG, PME, } \\
\text { PE, PL, GL, PIM }\end{array}$ & PIII & $\begin{array}{c}\text { PIII; PME, } \\
\text { PC, DPG, } \\
\text { PG, PL1, PL2 }\end{array}$ \\
\hline Major menaquinones & $\begin{array}{l}\left.\text { MK-11( } \mathrm{H}_{4}\right), \\
\left.\text { MK-11( } \mathrm{H}_{6}\right) \\
\left.\text { MK-11( } \mathrm{H}_{8}\right)\end{array}$ & $\begin{array}{c}\text { MK-11, } \\
\left.\text { MK-11( } \mathrm{H}_{2}\right), \\
\left.\text { MK-11( } \mathrm{H}_{4}\right)\end{array}$ & $\begin{array}{r}\left.\text { MK-11( } \mathrm{H}_{6}\right) \\
\mathrm{MK}-11\left(\mathrm{H}_{8}\right)\end{array}$ & $\begin{array}{c}\text { MK-10, } \\
\text { MK-10 }\left(\mathrm{H}_{2}\right) \\
\left.\text { MK-10( } \mathrm{H}_{4}\right)\end{array}$ & $\begin{array}{l}\text { MK-9 }\left(\mathrm{H}_{4}\right) \\
\text { MK-10( }\left(\mathrm{H}_{2}\right), \\
\left.\text { MK-10( } \mathrm{H}_{4}\right) \\
\left.\text { MK-10( } \mathrm{H}_{6}\right)\end{array}$ & $\begin{array}{r}\text { MK-10 }\left(\mathrm{H}_{6}\right) ; \\
\text { MK-10( }\left(\mathrm{H}_{8}\right)\end{array}$ & $\begin{array}{c}\text { MK-10 }\left(\mathrm{H}_{8}\right), \\
\left.\text { MK-11( } \mathrm{H}_{8}\right), \\
\text { MK-10 }\left(\mathrm{H}_{6}\right), \\
\text { MK-12 }\end{array}$ \\
\hline Major cellular fatty acid $(>10 \%)$ & $\begin{array}{c}\text { iso } \mathrm{G} \mathrm{C}_{16: 1} \\
(21 \cdot 69 \%) \text {, TBSA } \\
(36 \cdot 51 \%) \\
\left(30^{\circ} \mathrm{C}\right)\end{array}$ & $\begin{array}{c}\text { iso- }_{16: 0} \\
(30 \cdot 7 \%), \\
\text { anteiso- } \mathrm{C}_{17: 0} \\
(10 \cdot 61 \%), \text { TBSA } \\
(21 \cdot 87 \%) \\
\left(37^{\circ} \mathrm{C}\right)\end{array}$ & $\begin{array}{c}\text { iso- } \mathrm{C}_{16: 0} \\
(30 \cdot 77 \%), \\
\text { anteiso-C } 17: 0 \\
(11 \cdot 21 \%), \text { TBSA } \\
(12 \cdot 17 \%) \\
\left(37^{\circ} \mathrm{C}\right)\end{array}$ & $\begin{array}{c}\text { iso- } \mathrm{C}_{16: 0} \\
(26 \cdot 02 \%), \\
\text { anteiso-C } 17: 0 \\
(10 \cdot 07 \%), \text { TBSA } \\
(29 \cdot 38 \%) \\
\left(37^{\circ} \mathrm{C}\right)\end{array}$ & $\begin{array}{c}\text { iso- } \mathrm{C}_{16: 0} \\
(24 \cdot 17 \%), \\
\text { anteiso- } \mathrm{C}_{17: 0} \\
(13 \cdot 64 \%), \text { TBSA } \\
(33 \cdot 19 \%) \\
\left(37^{\circ} \mathrm{C}\right)\end{array}$ & NT & $\begin{array}{c}\text { iso- } \mathrm{C}_{16: 0} \\
(16 \cdot 0 \%), \\
\text { anteiso- } \mathrm{C}_{15: 0} \\
(18 \cdot 9 \%), \\
\text { anteiso- } \mathrm{C}_{17: 0} \\
(12 \cdot 8 \%)\left(28^{\circ} \mathrm{C}\right)\end{array}$ \\
\hline DNA G $+\mathrm{C}$ content $(\mathrm{mol} \%)$ & $68 \cdot 1$ & $67 \cdot 9$ & $69 \cdot 0$ & $71 \cdot 8$ & $73 \cdot 2$ & $71 \cdot 5$ & $74 \cdot 7$ \\
\hline
\end{tabular}

PIII refers to the phospholipid pattern as described by Lechevalier et al. (1977). 


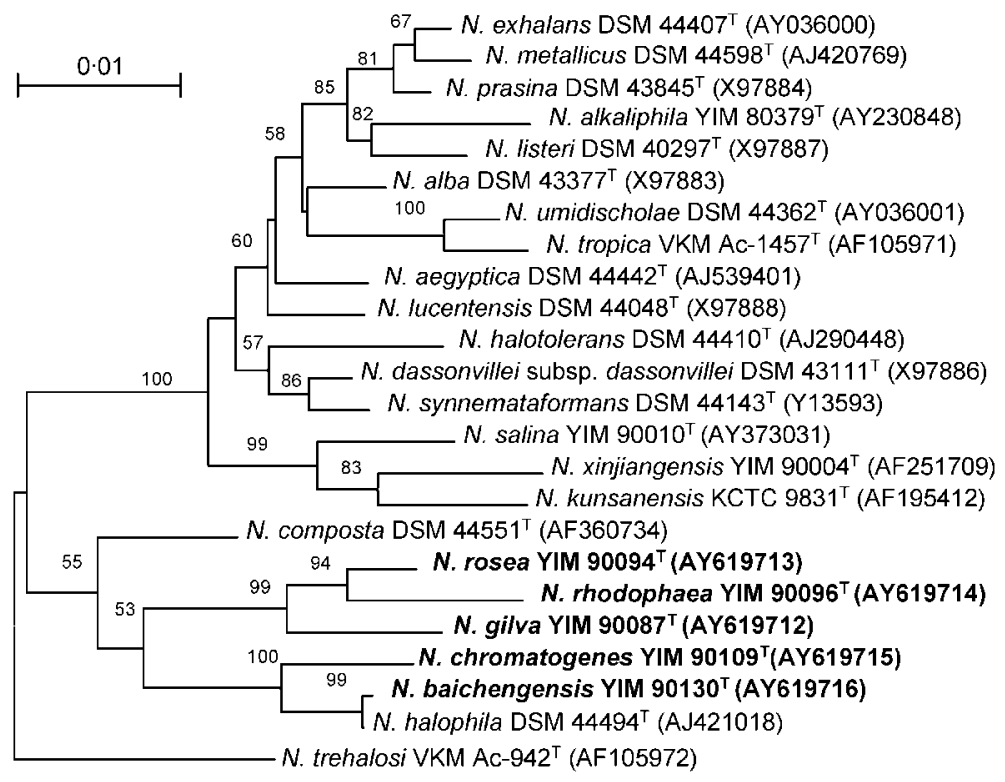

Fig. 1. Phylogenetic dendrogram obtained by distance matrix analysis of $16 \mathrm{~S}$ rRNA gene sequences showing the position of strains YIM $90087^{\top}$, YIM $90094^{\top}$, YIM $90096^{\top}$, YIM 90109 ${ }^{\top}$ and YIM 90130 amongst their phylogenetic neighbours. Numbers at branch nodes are bootstrap values (1000 resamplings). The gene sequence of Actinomadura madurae DSM $43067^{\top}$ (X97889) was used as the root (not shown). Bar, $1 \%$ sequence divergence.

the DNA-DNA relatedness values were lower than $70 \%$, which is the recommended threshold value for the delineation of genomic species (Wayne et al., 1987). On the basis of these results, the novel isolates represent five novel species of the genus Nocardiopsis.

Additionally, the five novel isolates could be distinguished from each other and from the two closely related Nocardiopsis species $N$. halophila and $N$. composta on the basis of physiological and biochemical characteristics and on chemotaxonomic data (Table 2).

Based on the results of phenotypic and genotypic analyses, we consider that the novel isolates represent five novel species of the genus Nocardiopsis, for which we propose the names Nocardiopsis gilva sp. nov., Nocardiopsis rosea sp. nov., Nocardiopsis rhodophaea sp. nov., Nocardiopsis chromatogenes sp. nov. and Nocardiopsis baichengensis sp. nov.

\section{Emended description of Nocardiopsis salina Li et al. 2004}

The description is the same as that given by Li et al. (2004) except for polar lipid and menaquinone compositions. In addition to PI and PG, the type strain also contains PC, DPG, phosphatidylethanolamine (PE), PME and four small phospholipid spots above DPG of unknown structure which are diagnostic for Nocardiopsis strains. The menaquinone pattern is mainly composed of MK-9 $\left(\mathrm{H}_{8}\right)$ and MK-10 $\left(\mathrm{H}_{8}\right)$ and smaller amounts of MK-9 $\left(\mathrm{H}_{4}\right), \mathrm{MK}-9\left(\mathrm{H}_{6}\right), \mathrm{MK}-10\left(\mathrm{H}_{4}\right)$, $\mathrm{MK}-10\left(\mathrm{H}_{6}\right)$ and $\mathrm{MK}-11\left(\mathrm{H}_{4}\right)$.

\section{Description of Nocardiopsis gilva sp. nov.}

Nocardiopsis gilva (gil'va. L. fem. adj. gilva pale yellow).
Aerobic, Gram-positive, non-acid-fast, non-motile organism. Aerial mycelium is pale yellow to yellow-white and the substrate mycelium is pale yellow to pale greenish-yellow on media tested. No diffusible pigments are produced. Vegetative hyphae are well developed and fragmented. Spiral spore chains are short and are borne on the aerial hyphae. Spores are smooth surfaced and non-motile. Optimal growth at 28-30 ${ }^{\circ} \mathrm{C}$ and at $\mathrm{pH} 7 \cdot 2$ with $5-8 \% \mathrm{NaCl}$. Temperature, $\mathrm{pH}$ and $\mathrm{NaCl}$ tolerance ranges are $10-40{ }^{\circ} \mathrm{C}, \mathrm{pH} 6 \cdot 0-9 \cdot 0$ and $0-18 \%(\mathrm{w} / \mathrm{v})$, respectively. L-Arabinose, D-cellobiose, D-fructose, D-galactose, D-glucose, glycerol, myo-inositol, D-lactose, D-mannitol, D-raffinose, sodium acetate, sodium citrate, D-sorbitol, starch, sucrose and D-xylose can be utilized as carbon sources, while D-maltose, D-mannose, L-rhamnose, D-ribose and D-xylitol can not be utilized. Alanine, arginine, asparagine, glycine, histidine, lysine, proline and threonine can be used as sole nitrogen sources, but adenine, cystine, glutamic acid, hydroxyproline, methionine, phenylalanine, tryptophan and valine can not be utilized. Milk coagulation, milk peptonization, gelatin liquefaction, starch hydrolysis, $\mathrm{H}_{2} \mathrm{~S}$ production, urease activity and melanin production are negative, but nitrate reduction is positive. Cell walls contain meso-diaminopimelic acid as the diagnostic diamino acid and have no diagnostic sugar. The polar lipid pattern is composed of PME, PC, PI, PG and DPG together with some unknown PGL and unknown PL. Major menaquinones are MK$11\left(\mathrm{H}_{4}\right), \mathrm{MK}-11\left(\mathrm{H}_{6}\right)$ and $\mathrm{MK}-11\left(\mathrm{H}_{8}\right)$. The cellular fatty acid profile contains iso- $\mathrm{C}_{16: 1}(21 \cdot 7 \%), \mathrm{C}_{18: 0}$ 10-methyl $(36.5 \%)$, iso- $\mathrm{C}_{14: 0}(0 \cdot 7 \%)$, iso- $\mathrm{C}_{15: 0}(1 \cdot 1 \%)$, anteiso$\mathrm{C}_{15: 0}(2 \cdot 1 \%)$, iso- $\mathrm{C}_{16: 0}(6 \cdot 6 \%), \mathrm{C}_{16: 0}(1 \cdot 3 \%), \mathrm{C}_{16: 0} 10-$ methyl $(3 \cdot 7 \%)$, anteiso- $\mathrm{C}_{17: 1}(8 \cdot 5 \%)$, iso- $\mathrm{C}_{17: 0}(1 \cdot 8 \%)$, anteiso- $\mathrm{C}_{17: 0}(3 \cdot 6 \%), \mathrm{C}_{17: 0} 10$-methyl $(3 \cdot 9 \%)$, iso- $\mathrm{C}_{18: 0}$ $(4 \cdot 2 \%), \quad \mathrm{C}_{18: 1} \omega 9 c(1.5 \%)$, anteiso- $\mathrm{C}_{19: 0}(0.6 \%)$ and $\mathrm{C}_{16: 1} \omega 7 c(2 \cdot 0 \%)$. DNA G $+\mathrm{C}$ content is $68 \cdot 1 \mathrm{~mol} \%$. 
The type strain, YIM $90087^{\mathrm{T}}\left(=\right.$ KCTC $19006^{\mathrm{T}}=$ CCTCC AA $2040012^{\mathrm{T}}=\mathrm{DSM} 44841^{\mathrm{T}}$ ), was isolated from a saline soil sample in the west of China.

\section{Description of Nocardiopsis rosea sp. nov.}

Nocardiopsis rosea (ro'se.a. L. fem. adj. rosea rose coloured).

Aerobic, Gram-positive, non-acid-fast, non-motile organism. Aerial mycelium is pink-white to pale pink and the substrate mycelium is pale pink to moderate red on media tested. No diffusible pigments are produced. Vegetative hyphae are well developed and fragmented. Spore chains are borne on the aerial hyphae. Spores are smooth surfaced and non-motile. Optimum growth at $37-40{ }^{\circ} \mathrm{C}$ and at $\mathrm{pH} 7 \cdot 2$ with $5-8 \% \mathrm{NaCl}$. Temperature, $\mathrm{pH}$ and $\mathrm{NaCl}$ tolerance ranges are $20-60{ }^{\circ} \mathrm{C}, \mathrm{pH} 6 \cdot 0-9 \cdot 0$ and $0-18 \%(\mathrm{w} / \mathrm{v})$, respectively. L-Arabinose, D-fructose, D-glucose, D-lactose, Dmaltose, L-rhamnose, D-ribose, sodium acetate, sucrose and starch can be utilized as carbon sources, but D-cellobiose, D-galactose, glycerol, myo-inositol, D-mannitol, D-mannose, D-raffinose, sodium citrate, D-sorbitol, D-xylitol and Dxylose can not be utilized. Alanine, arginine, asparagine, glycine, histidine and proline can be used as sole nitrogen sources, but adenine, cystine, glutamic acid, hydroxyproline, lysine, methionine, phenylalanine, threonine, tryptophan and valine can not be utilized. Milk coagulation, milk peptonization, gelatin liquefaction, starch hydrolysis, $\mathrm{H}_{2} \mathrm{~S}$ production, urease activity and melanin production are negative; nitrate reduction is positive. Cell walls contain meso-diaminopimelic acid as the diagnostic diamino acid and have no diagnostic sugar. The polar lipid pattern is composed of PME, PC, PI, PG and DPG together with some unknown PGL and unknown PL. Major menaquinones are MK-11, MK-11 $\left(\mathrm{H}_{2}\right)$ and MK-11 $\left(\mathrm{H}_{4}\right)$. The cellular fatty acid profile contains iso- $\mathrm{C}_{16: 0}(30 \cdot 7 \%)$, anteiso- $\mathrm{C}_{17: 0}(10 \cdot 6 \%)$, $\mathrm{C}_{18: 0} 10$-methyl $(21 \cdot 9 \%)$, iso- $\mathrm{C}_{10: 0}(1 \cdot 7 \%), \mathrm{C}_{10: 0}(0 \cdot 2 \%)$, anteiso- $\mathrm{C}_{11: 0}(0 \cdot 2 \%)$, iso- $\mathrm{C}_{14: 0}(1 \cdot 4 \%)$, iso- $\mathrm{C}_{15: 0}(0 \cdot 6 \%)$, anteiso- $\mathrm{C}_{15: 0}(2 \cdot 2 \%)$, iso- $\mathrm{C}_{16: 1}(1 \cdot 3 \%), \mathrm{C}_{16: 0}(1 \cdot 7 \%)$, $\mathrm{C}_{16: 0}$ 10-methyl (1.6\%), anteiso-A- $\mathrm{C}_{17: 1}(0.9 \%)$, iso- $\mathrm{C}_{17: 0}$ $(2 \cdot 7 \%), \mathrm{C}_{17: 0}(1 \cdot 2 \%), \mathrm{C}_{17: 0} 10$-methyl $(3.9 \%)$, iso- $\mathrm{C}_{18: 0}$ $(6 \cdot 7 \%), \mathrm{C}_{18: 1} \omega 9 c(0 \cdot 7 \%), \mathrm{C}_{18: 0}(8 \cdot 7 \%)$, anteiso- $\mathrm{C}_{19: 0}$ $(0 \cdot 5 \%), \mathrm{C}_{19: 0}(0 \cdot 5 \%)$ and $\mathrm{C}_{16: 1} \omega 7 c(0 \cdot 3 \%)$. DNA G +C content is $67 \cdot 9 \mathrm{~mol} \%$.

The type strain, YIM $90094^{\mathrm{T}}\left(=\right.$ KCTC $19007^{\mathrm{T}}=$ CCTCC AA $2040013^{\mathrm{T}}=\mathrm{DSM} 44842^{\mathrm{T}}$ ), was isolated from a saline soil sample in the west of China.

\section{Description of Nocardiopsis rhodophaea sp. nov.}

Nocardiopsis rhodophaea [rho.do.phae'a. Gr. n. rhodos the rose; Gr. adj. phaeos brown; N.L. fem. adj. rhodophaea rosebrown (after the colour of the substrate mycelium)].

Aerobic, Gram-positive, non-acid-fast, non-motile organism. Aerial mycelium is pale pink to light reddish brown and the substrate mycelium is light reddish brown to deep reddish brown on media tested. No diffusible pigments are produced. Vegetative hyphae are well developed and fragmented. Short spore chains are borne on the aerial hyphae. Spores are smooth surfaced and non-motile. Optimum growth at $37-40{ }^{\circ} \mathrm{C}$ and at $\mathrm{pH} 7 \cdot 2$ with $5-8 \% \mathrm{NaCl}$. Temperature, $\mathrm{pH}$ and $\mathrm{NaCl}$ tolerance ranges are $20-60{ }^{\circ} \mathrm{C}$, $\mathrm{pH} 6 \cdot 0-9 \cdot 0$ and $0-18 \%(\mathrm{w} / \mathrm{v})$, respectively. L-Arabinose, D-glucose, glycerol, myo-inositol, sodium acetate and D-ribose can be utilized as carbon sources, but D-cellobiose, D-fructose, D-galactose, D-lactose, D-maltose, D-mannose, D-mannitol, D-raffinose, L-rhamnose, sodium citrate, Dsorbitol, starch, sucrose, D-xylitol and D-xylose can not be utilized. Alanine, arginine, asparagine, glycine, histidine, proline and valine are used as sole nitrogen sources, while adenine, cystine, glutamic acid, hydroxyproline, lysine, methionine, phenylalanine, threonine and tryptophan are not be utilized. Milk coagulation, gelatin liquefaction, starch hydrolysis, $\mathrm{H}_{2} \mathrm{~S}$ production, urease activity, nitrate reduction and melanin production are negative, but milk peptonization is positive. Cell walls contain meso-diaminopimelic acid as the diagnostic diamino acid and have no diagnostic sugars. The polar lipid pattern is composed of PME, PC, PI, PG, DPG and PIM, together with some unknown PGL and unknown PL. Major menaquinones are MK-11 $\left(\mathrm{H}_{6}\right)$ and MK-11 $\left(\mathrm{H}_{8}\right)$. The cellular fatty acid profile contains iso- $\mathrm{C}_{16: 0}(30 \cdot 8 \%)$, anteiso- $\mathrm{C}_{17: 0}(11 \cdot 2 \%), \mathrm{C}_{18: 0}$ 10 -methyl $(12 \cdot 2 \%)$, iso- $\mathrm{C}_{14: 0}(1 \cdot 6 \%)$, iso- $\mathrm{C}_{15: 0}(2 \cdot 4 \%)$, anteiso- $\mathrm{C}_{15: 0}(5 \cdot 8 \%)$, iso- $\mathrm{C}_{16: 1}(1 \cdot 3 \%), \mathrm{C}_{16: 0}(2 \cdot 0 \%)$, $\mathrm{C}_{16: 0}$ 10-methyl (1.3\%), iso- $\mathrm{C}_{17: 0}(4 \cdot 1 \%), \mathrm{C}_{17: 1} \omega 8 \mathrm{c}$ $(0.9 \%), \mathrm{C}_{17: 0}(1 \cdot 1 \%), \mathrm{C}_{17: 0} 10$-methyl $(8.9 \%)$, iso- $\mathrm{C}_{18: 0}$ $(4 \cdot 8 \%), \mathrm{C}_{18: 1} \omega 9 c(3 \cdot 4 \%), \mathrm{C}_{18: 0}(7 \cdot 1 \%)$ and $\mathrm{C}_{16: 1} \omega 7 c$ $(1 \cdot 2 \%)$. DNA G + C content is $69 \cdot 0 \mathrm{~mol} \%$.

The type strain, YIM $90096^{\mathrm{T}}\left(=\right.$ KCTC $19049^{\mathrm{T}}=$ CCTCC AA $2040014^{\mathrm{T}}=\mathrm{DSM} 44843^{\mathrm{T}}$ ), was isolated from a saline soil sample in the west of China.

\section{Description of Nocardiopsis chromatogenes sp. nov.}

Nocardiopsis chromatogenes (chro'ma.to.gen.es. Gr. n. chroma -atos colour; Gr. v. gennaio to produce; N.L. part. adj. chromatogenes producing colour).

Aerobic, Gram-positive, non-acid-fast, non-motile organism. Aerial mycelium is white and the substrate mycelium is light reddish brown to deep reddish brown on most media tested. Yellowish pink pigments are produced on Czapek agar and inorganic salts-starch agar (ISP4) media. Vegetative hyphae are well developed and fragmented. Long spore chains are borne on the aerial hyphae. Spores are smooth surfaced and non-motile. Optimum growth at $37-40^{\circ} \mathrm{C}$ and at $\mathrm{pH} 7 \cdot 2$ with $5-8 \% \mathrm{NaCl}$. Temperature, $\mathrm{pH}$ and $\mathrm{NaCl}$ tolerance ranges are $20-60{ }^{\circ} \mathrm{C}, \mathrm{pH} 6 \cdot 0-9 \cdot 0$ and $0-18 \%$ $(\mathrm{w} / \mathrm{v})$, respectively. L-Arabinose, D-cellobiose, D-fructose, D-galactose, D-glucose, glycerol, myo-inositol, D-lactose, D-maltose, D-mannitol, D-mannose, D-raffinose, L-rhamnose, D-ribose, sodium acetate, sodium citrate, sucrose and D-xylose can be utilized as carbon sources, but D-sorbitol, starch and D-xylitol can not be utilized. Alanine, arginine, 
asparagine, glycine, histidine, lysine, phenylalanine, proline, threonine, tryptophan and valine can be used as sole nitrogen sources, but adenine, cystine, glutamic acid, hydroxyproline and methionine can not be utilized. Milk coagulation, milk peptonization, gelatin liquefaction, nitrate reduction, $\mathrm{H}_{2} \mathrm{~S}$ production and urease activity are negative, but starch hydrolysis and melanin production are positive. Cell walls contain meso-diaminopimelic acid as the diagnostic diamino acid and have no diagnostic sugars. The polar lipid pattern is composed of PME, PC, PI, PG, DPG and PIM, together with some unknown PGL and unknown PL. Major menaquinones are MK-10, MK-10 $\left(\mathrm{H}_{2}\right)$ and MK$10\left(\mathrm{H}_{4}\right)$. The cellular fatty acid profile contains iso- $\mathrm{C}_{16: 0}$ $(26 \cdot 0 \%)$, anteiso- $\mathrm{C}_{17: 0}(10 \cdot 1 \%), \mathrm{C}_{18: 0} 10$-methyl $(29 \cdot 4 \%)$, iso- $\mathrm{C}_{10: 0}(1 \cdot 3 \%), \mathrm{C}_{10: 0}(1 \cdot 0 \%)$, anteiso- $\mathrm{C}_{11: 0}(0 \cdot 7 \%)$, iso$\mathrm{C}_{14: 0}(3 \cdot 0 \%)$, iso- $\mathrm{C}_{15: 0}(0 \cdot 8 \%)$, anteiso- $\mathrm{C}_{15: 0}(7 \cdot 3 \%)$, i-C $16: 1(2 \cdot 2 \%), \mathrm{C}_{16: 0}(2 \cdot 8 \%), \mathrm{C}_{16: 0} 10$-methyl $(1 \cdot 7 \%)$, anteiso-A- $\mathrm{C}_{17: 1}(1 \cdot 5 \%), \mathrm{C}_{17: 0} 10$-methyl $(2 \cdot 2 \%)$, iso- $\mathrm{C}_{18: 0}$ $(1 \cdot 9 \%), \mathrm{C}_{18: 0}(5 \cdot 6 \%)$ and $\mathrm{C}_{16: 1} \omega 7 c(1 \cdot 0 \%)$. DNA G $+\mathrm{C}$ content is $71 \cdot 8 \mathrm{~mol} \%$.

The type strain, YIM $90109^{\mathrm{T}}\left(=\right.$ KCTC $19008^{\mathrm{T}}=$ CCTCC AA $2040015^{\mathrm{T}}=\mathrm{DSM} 44844^{\mathrm{T}}$ ), was isolated from a saline soil sample in the west of China.

\section{Description of Nocardiopsis baichengensis sp. nov.}

Nocardiopsis baichengensis (bai.cheng.en'sis. N.L. fem. adj. baichengensis pertaining to Baicheng, a county of Xinjiang Province in the west of China where the type strain was collected).

Aerobic, Gram-positive, non-acid-fast, non-motile organism. Aerial mycelium is white to yellow-white and the substrate mycelium is light yellow to deep orange-yellow on the media tested. No diffusible pigments are produced. Vegetative hyphae are well developed and fragmented. Long spore chains are borne on the aerial hyphae. Spores are smooth surfaced and non-motile. Optimum growth at $37-40{ }^{\circ} \mathrm{C}$ and at $\mathrm{pH} 7 \cdot 2$ with $5-8 \% \mathrm{NaCl}$. Temperature, $\mathrm{pH}$ and $\mathrm{NaCl}$ tolerance ranges are $20-50^{\circ} \mathrm{C}, \mathrm{pH} 6 \cdot 0-9 \cdot 0$ and $0-18 \%(\mathrm{w} / \mathrm{v})$, respectively. L-Arabinose, D-cellobiose, D-fructose, D-galactose, D-glucose, glycerol, D-maltose, D-mannitol, D-mannose, L-rhamnose, D-ribose, sodium acetate, sodium citrate and sucrose can be utilized as carbon sources, but myo-inositol, D-lactose, D-raffinose, D-sorbitol, starch, D-xylitol and D-xylose can not be utilized. Alanine, arginine, asparagine, glycine, histidine, lysine, phenylalanine, proline, threonine, tryptophan and valine can be used as sole nitrogen sources, but adenine, cystine, glutamic acid, hydroxyproline and methionine can not be utilized. Milk coagulation, milk peptonization, starch hydrolysis, $\mathrm{H}_{2} \mathrm{~S}$ production, nitrate reduction and urease activity are negative, but gelatin liquefaction and melanin production are positive. Cell walls contain meso-diaminopimelic acid as the diagnostic diamino acid and have no diagnostic sugars. The polar lipid pattern is composed of PME, PC, PI, PG, DPG and PIM, together with some unknown PGL and unknown PL. Major menaquinones are MK-10 $\left(\mathrm{H}_{2}\right)$, MK$10\left(\mathrm{H}_{4}\right)$ and MK-10 $\left(\mathrm{H}_{6}\right)$. The cellular fatty acid profile contains iso- $\mathrm{C}_{16: 0}(24 \cdot 2 \%)$, anteiso- $\mathrm{C}_{17: 0}(13 \cdot 6 \%), \mathrm{C}_{18: 0}$ 10 -methyl $(33 \cdot 2 \%)$, iso- $\mathrm{C}_{14: 0}(1 \cdot 0 \%), \mathrm{C}_{14: 0}(0 \cdot 3 \%)$, iso$\mathrm{C}_{15: 0}(0 \cdot 5 \%)$, anteiso- $\mathrm{C}_{15: 0}(3 \cdot 8 \%), \mathrm{i}-\mathrm{C}_{16: 1}(1 \cdot 7 \%), \mathrm{C}_{16: 0}$ $(2 \cdot 8 \%), \mathrm{C}_{16: 0} 10$-methyl $(1 \cdot 7 \%)$, anteiso-A-C $\mathrm{C}_{17: 1}(1 \cdot 9 \%)$, iso- $\mathrm{C}_{17: 0}(1 \cdot 1 \%), \mathrm{C}_{17: 0}(0 \cdot 3 \%), \mathrm{C}_{17: 0} 10$-methyl $(1 \cdot 7 \%)$, iso- $\mathrm{C}_{18: 0}(3 \cdot 5 \%), \mathrm{C}_{18: 1} \omega 9 c(0 \cdot 5 \%), \mathrm{C}_{18: 0}(6 \cdot 1 \%)$, anteiso$\mathrm{C}_{19: 0}(0 \cdot 5 \%)$ and $\mathrm{C}_{16: 1} \omega 7 c(1 \cdot 5 \%)$. DNA G $+\mathrm{C}$ content is $73 \cdot 2 \mathrm{~mol} \%$.

The type strain, YIM $90130^{\mathrm{T}}\left(=\mathrm{KCTC} 19009^{\mathrm{T}}=\right.$ CCTCC AA $2040016^{\mathrm{T}}=\mathrm{DSM} 44845^{\mathrm{T}}$ ), was isolated from a saline soil sample in the west of China.

\section{Acknowledgements}

The authors are grateful to Professor Dr Hans G. Trüper for the Latin construction of the species epithets. This research was supported by National Basic Research Program of China (project no. 2004CB719601), National Natural Science Foundation of China (project no. 30270004), Yunnan Provincial Natural Science Foundation (project no. 2004C0002Q) and the 21C Frontier Microbial Genomics and Application Center Program, Minister of Science and Technology (MG05-0101-1-0) and the International Cooperation R and D Program, Minister of Science and Technology (M6-0203-000002), Korea. W. -J.L. was also supported by the Program for New Century Excellent Talent in University (NCET).

\section{References}

Al-Tai, A. M. \& Ruan, J.-S. (1994). Nocardiopsis halophila sp. nov., a new halophilic actinomycete isolated from soil. Int J Syst Bacteriol 44, 474-478.

Al-Zarban, S. S., Abbas, I., Al-Musallam, A. A., Steiner, U., Stackebrandt, E. \& Kroppenstedt, R. M. (2002). Nocardiopsis halotolerans sp. nov., isolated from salt marsh soil in Kuwait. Int J Syst Evol Microbiol 52, 525-529.

Chun, J., Bae, K.-S., Moon, E.-Y., Jung, S.-O., Lee, H.-K. \& Kim, S.-J. (2000). Nocardiopsis kunsanensis sp. nov., a moderately halophilic actinomycete isolated from a saltern. Int J Syst Evol Microbiol 50, 1909-1913.

Cui, X. L., Mao, P. H., Zeng, M., Li, W. J., Zhang, L. P., Xu, L. H. \& Jiang, C. L. (2001). Streptimonospora salina gen. nov., sp. nov., a new member of the family Nocardiopsaceae. Int J Syst Evol Microbiol 51, 357-363.

De Ley, J., Cattoir, H. \& Reynaerts, A. (1970). The quantitative measurement of DNA hybridization from renaturation rates. Eur $J$ Biochem 12, 133-142.

Felsenstein, J. (1985). Confidence limits on phylogenies: an approach using the bootstrap. Evolution 39, 783-791.

Felsenstein, J. (1993). PHYLIP (phylogeny inference package), version 3.5c. Distributed by the author. Department of Genome Sciences, University of Washington, Seattle, USA.

Hasegawa, T., Takizawa, M. \& Tanida, S. (1983). A rapid analysis for chemical grouping aerobic actinomycetes. J Gen Appl Microbiol 29, 319-322.

Hozzein, W. N., Li, W.-J., Ali, M. I. A., Hammouda, O., Mousa, A. S., Xu, L.-H. \& Jiang, C.-L. (2004). Nocardiopsis alkaliphila sp. nov., a novel alkaliphilic actinomycete isolated from desert soil in Egypt. Int J Syst Evol Microbiol 54, 247-252. 
Huß, V. A. R., Festl, H. \& Schleifer, K. H. (1983). Studies on the spectrophotometric determination of DNA hybridization from renaturation rates. Syst Appl Microbiol 4, 184-192.

Jahnke, K.-D. (1992). BASIC computer program for evaluation of spectroscopic DNA renaturation data from Gilford System 2600 spectrophotometer on a PC/XT/AT type personal computer. J Microbiol Methods 15, 61-73.

Kämpfer, P., Busse, H.-J. \& Rainey, F. A. (2002). Nocardiopsis compostus sp. nov., from the atmosphere of a composting facility. Int J Syst Evol Microbiol 52, 621-627.

Kelly, K. L. (1964). Inter-Society Color Council-National Bureau of Standards Color Name Charts Illustrated with Centroid Colors. Washington, DC: US Government Printing Office.

Kimura, M. (1980). A simple method for estimating evolutionary rates of base substitutions through comparative studies of nucleotide sequences. J Mol Evol 16, 111-120.

Kimura, M. (1983). The Neutral Theory of Molecular Evolution. Cambridge: Cambridge University Press.

Kroppenstedt, R. M. (1982). Separation of bacterial menaquinones by HPLC using reverse phase (RP 18) and a silver loaded ion exchanger as stationary phases. J Liquid Chromatogr 5, 2359-2387.

Kumar, S., Tamura, K., Jakobsen, I. B. \& Nei, M. (2001). MEGA2: molecular evolutionary genetics analysis software. Bioinformatics 17, 1244-1245.

Lechevalier, M. P., De Bièvre, C. \& Lechevalier, H. A. (1977). Chemotaxonomy of aerobic actinomycetes: phospholipid composition. Biochem Syst Ecol 5, 249-260.

Li, M.-G., Li, W.-J., Xu, P., Cui, X.-L., Xu, L.-H. \& Jiang, C.-L. (2003). Nocardiopsis xinjiangensis sp. nov., a halophilic actinomycete isolated from saline soil sample in China. Int J Syst Evol Microbiol 53, 317-321.

Li, W.-J., Park, D.-J., Tang, S.-K., Wang, D., Lee, J.-C., Xu, L.-H., Kim, C.-J. \& Jiang, C.-L. (2004). Nocardiopsis salina sp. nov., a novel halophilic actinomycete isolated from a saline soil in China. Int J Syst Evol Microbiol 54, 1805-1809.

Locci, R. (1989). Streptomycetes and related genera. In Bergey's Manual of Systematic Bacteriology, vol. 4, pp. 2451-2508. Edited by S. T. Williams, M. E. Sharpe \& J. G. Holt. Baltimore: Williams \& Wilkins.

Marmur, J. (1961). A procedure for the isolation of deoxyribonucleic acid from microorganisms. J Mol Biol 3, 208-218.
Marmur, J. \& Doty, P. (1962). Determination of the base composition of deoxyribonucleic acid from its thermal denaturation temperature. J Mol Biol 5, 109-118.

Meyer, J. (1976). Nocardiopsis, a new genus of the order Actinomycetales. Int J Syst Bacteriol 26, 487-493.

Minnikin, D. E., O'Donnell, A. G., Goodfellow, M., Alderson, G., Athalye, M., Schaal, A. \& Parlett, J. H. (1984). An integrated procedure for the extraction of isoprenoid quinines and polar lipids. J Microbiol Methods 2, 233-241.

Sabry, S. A., Ghanem, N. B., Abu-Ella, G. A., Schumann, P., Stackebrandt, E. \& Kroppenstedt, R. M. (2004). Nocardiopsis aegyptia sp. nov., isolated from marine sediment. Int J Syst Evol Microbiol 54, 453-456.

Saitou, N. \& Nei, M. (1987). The neighbor-joining method: a new method for reconstructing phylogenetic trees. Mol Biol Evol 4, 406-425.

Sasser, M. (1990). Identification of bacteria by gas chromatography of cellular fatty acids. USFCC Newsl 20, 16.

Shirling, E. B. \& Gottlieb, D. (1966). Methods for characterization of Streptomyces species. Int J Syst Bacteriol 16, 313-340.

Stackebrandt, E. \& Goebel, B. M. (1994). Taxonomic note: a place for DNA-DNA reassociation and 16S rRNA sequence analysis in the present species definition in bacteriology. Int J Syst Bacteriol 44, 846-849.

Tang, S.-K., Li, W.-J., Wang, D., Zhang, Y.-G., Xu, L.-H. \& Jiang, C.-L. (2003). Studies of the biological characteristics of some halophilic and halotolerant actinomycetes isolated from saline and alkaline soils. Actinomycetologica 17, 6-10.

Thompson, J. D., Gibson, T. J., Plewniak, F., Jeanmougin, F. \& Higgins, D. G. (1997). The CLUSTAL_X windows interface: flexible strategies for multiple sequence alignment aided by quality analysis tools. Nucleic Acids Res 25, 4876-4882.

Wayne, L. G., Brenner, D. J., Colwell, R. R. \& 9 other authors (1987). International Committee on Systematic Bacteriology. Report of the ad hoc committee on reconciliation of approaches to bacterial systematics. Int J Syst Bacteriol 37, 463-464.

Xu, P., Li, W.-J., Xu, L.-H. \& Jiang, C.-L. (2003). A microwave based method for genomic DNA extraction from Actinomycetes. Microbiology (Beijing) 30, 82-84 (in Chinese). 